Bangladesh J. Zool. 48(2): 413-428, 2020

ISSN: 0304-9027

eISSN: $2408-8455$

\title{
BUTTERFLY DIVERSITY IN THE THREE SELECTED AREAS IN DHAKA CITY, BANGLADESH
}

\author{
Md. Aminul Islam ${ }^{1 *}$, Md. Abul Kashem², Md. Abdul Alim², Fatema-Tuz-Zohora² \\ Department of Zoology, University of Dhaka, Dhaka, Bangladesh
}

\begin{abstract}
The diversity of butterfly species was studied from January to June 2015 in the three selected areas, viz. Ramna Park, Jagannath University Campus, and Baldha Garden in Dhaka city, Bangladesh. A total 75 species of butterfly belonging to 42 genera under 8 families were recorded from the study areas during the study period. Of them, 52 species (6253 individuals) of 8 families were found in the Ramna Park, 37 species (1430 individuals) of 7 families in the Jagannath University Campus and 20 species (320 individuals) of 6 families in the Boldha Garden. In these three study areas, 17 species, 14 genera and 6 families were in common. At the Ramna Park, the highest species richness included the family Lycaenidae $(21.15 \%, 11 \mathrm{spp}$.) followed by Papilionidae (17.31\%, 9 spp.), Pieridae (17.31\%, 9 spp.), Nymphalidae $(17.31 \%$, 9 spp.), Hesperiidae (13.46\%, 7 spp.), Danaidae (7.69\%, 4 spp.), Satyridae $(3.85 \%, 2$ spp.), and the lowest was in the family Acriidae (1.92\%, 1 spp.). At the Jagannath University Campus the highest number of species were recorded in the family Lycaenidae $(21.62 \%, 8$ spp.) and Hesperiidae (21.62\%, 8 spp.) followed by Pieridae (18.92\%, 7 spp.), Papilionidae (13.51\%, 5 spp.), Nymphalidae (10.81\%, 4 spp.), Danaidae $(10.81 \%, 4$ spp.), and the lowest was in Satyridae $(2.70 \%, 1$ spp.). The topmost butterfly species were recorded in the family Pieridae (25\%, $5 \mathrm{spp}$.) followed by Lycaenidae (20\%, 4 spp.), Nymphalidae (20\%, 4 spp.), Papilionidae (15\%, 3 spp.), and the lowest from the family Hesperiidae (10\%, 2 spp.) and Danaidae (10\%, 2 spp.) at the Baldha Garden. The maximum butterfly species were found in February to June (51 spp.), February (34 spp.), and April (16 spp.), and the lowest were in January (46 spp.), June (21 spp.) and January (8 spp.), respectively for the Ramna Park, Jagannath University Campus and the Baldha Garden. The peak of the population observed in May ( $\mathrm{n}=1285)$, March $(\mathrm{n}=325)$, and May $(\mathrm{n}=71)$, and the lowest was in January $(n=662)$, June $(n=145)$ and January $(n=21)$, respectively for the Ramna Park, Jagannath University Campus, and Baldha Garden. The Shanon's Diversity Index $(\mathrm{H})$ and Simpson's Index $(\lambda)$ indicated high butterfly diversity at the Ramna Park $(\mathrm{H}=3.68, \lambda=0.03)$, Jagannath University Campus $(\mathrm{H}=3.20, \lambda=0.06)$ and Baldha Garden $(\mathrm{H}=2.50, \lambda=0.13)$, respectively. The high Species Evenness in the Ramna Park $(E=0.93)$, Jaganath University Campus $(E=0.89)$ and the Baldha Garden $(E=$ $0.84)$ indicated that the species were evenly distributed. The calculated Sorenson's Coefficient (CC) was 0.47 , which indicates that these three communities have quite a bit of overlap or similarity.

Key words: Butterfly Diversity, Diversity Index, Species Evenness, Community Similarity, Sorenson's Coefficient.
\end{abstract}

* Author for corresponding: <aminul.ek@du.ac.bd>; 2Department of Zoology, Jagannath University, Dhaka, Bangladesh.

(12020 Zoological Society of Bangladesh DOI: https://doi.org/10.3329 /bjz.v48i2.52380 


\section{INTRODUCTION}

Butterflies are scaled wing insects, which belong to the order Lepidoptera, class Insecta and phylum Arthropoda (Nimblkar et al. 2011). They are large and diverse group of animals that are a conspicuous part of virtually all the world's terrestrial ecosystems (Singh 2011). There are more than 17,500 butterflies species under 17 families in the world and $90 \%$ of them have been documented (Robbins and Olper 1997). Among them, 1501 species have been recorded from India, 651 species from Nepal, 242 species from Srilanka, 237 species from Japan, 1182 species from Malaysia (Gaonkar 1996). A total of 305 butterflies species under 10 families have been reported from Bangladesh (Bangladesh, I. U. C. N. 2015).

Butterflies have been regarded as the sign of beauty and elegance in nature (Rafi et al. 2000). They are important aspects of ecosystem for their interaction with plants as pollinators and herbivores. They occupy a vital position or disproportionate effect on other organisms in different ecosystems and their occurrence and diversity has been invoked as an indicator of biological diversity and good health (Aluri and Rao 2002, Schmucki et al. 2015). Butterflies are keystone species of environmental changes as they are sensitive to habitat degradation and climate change (Kunte 2000) due to their rapid life cycle than higher animals and hence can be quicker to react to small changes in their environment (Lafontaine 1997).

Butterfly distribution, diversity, and abundance depend upon the key factors such as the greatest diversity of plants, habitats, topography, and climates (Sarder et al. 2016). Butterfly species are generally abundant in tropical area means tropical forest, Shal forest, and tropical rainforest. In Bangladesh very insufficient and limited works have been done on butterfly species diversity, species composition and its distribution pattern (Bashar 2014). Hence, in various ecological pockets of primary and disturbed (human dominated) habitat of butterfly fauna, it requires proper exploration (Sarder et al. 2016). Many species of butterflies depend on relic vegetation for survival, especially in urban areas (Blair 1999). Dhaka is the capital of Bangladesh and its climate is very favorable for butterfly species diversity having a tropical Savanna climate. However, no detailed work has done on the diversity of butterfly in Dhaka city. The aim of the present study was to evaluate the diversity, species richness and evenness and community similarity of butterfly fauna of the three selected places of the Dhaka city. 


\section{MATERIAL AND METHODS}

Study area: The study was conducted in the following 3 selected areas in Dhaka city:

Ramna Park: It is located at the center of the Dhaka city. The geological coordinates are Latitude: $23^{\circ} 44^{\prime} 14.70^{\prime \prime} \mathrm{N}$ Longitude: $90^{\circ} 24^{\prime} 03.4^{\prime \prime} \mathrm{E}$. The total area is 68.50 acres. There are 71 species of flowering trees, shrubs, perennials, and annuals, 41 species of forestry, 36 species of fruit-bearing plants, 33 species of medicinal plants, and 11 other species.

Jagannath University Campus: It is located at the southern part of the Dhaka city. The geological co-ordinates are Latitude: $23^{\circ} 42^{\prime} 32.4^{\prime \prime} \mathrm{N}$ and Longitude: $90^{\circ} 24^{\prime} 38.16^{\prime \prime} \mathrm{E}$. Total area is 7.5 acres. It can be compared with the overall vegetation trend of the Dhaka city. Most of its areas are covered with the concrete buildings while the botanical garden, life, and earth science faculty are the oasis of this area. The variety of species stretches from shrubs to medicinal plant and flower-bearing trees.

Baldha Garden: It is located at the south eastern part of the Dhaka city. The geological co-ordinates are Latitude: $23^{\circ} 43^{\prime} 06^{\prime \prime} \mathrm{N}$ and Longitude: $90^{\circ} 25^{\prime} 04^{\prime \prime} \mathrm{E}$. It is 3.15 acres compact area with variety of vegetation. There is a wide variation of floral species occurred in the Baldha garden especially in the psyche section. There are about 600 species of plants from around the world cultured here.

Materials: Sweep net, camera, collecting jar and polythene bag, forceps and needle, envelopes, permanent ink pen, label paper and magnifying glass were used as physical materials. The biological materials were butterflies and the related plants.

Sampling and Identification: Butterflies were observed during sunny days at a constant speed from 7 am to $2 \mathrm{pm}$ (BST) four days in a month through walking transect (Caldas and Robbins 2003). Butterfly Identification was carried out by using the keys developed and used by Marshall de Niceville (1883), Bingham (1905), Evans (1932), Wynter-Blyth (1957), Talbot (1978), Bashar (2014), and Encyclopedia of fauna (Ahmed et al. 2009).

On the basis of abundance the observed butterflies were categorized in five categories in the three study areas. Species which observed a total of abundance exceeding 100 individuals were described as very common (VC: > 100 sightings), common (C: 50-100 sightings), not rare (NR: 15-49 sightings), rare (R: 3-14 sightings), very rare (VR: 1-2 sightings) (Nidup et al. 2014).

Diversity Analysis: The study used Shannon's Diversity Index (H) (Shannon and Weiner 1949) and Simpson's Index ( $\lambda$ ) (Simpson 1949) as models as a measure of diversity. The equations for the two indices: 
Shannon's Diversity Index $(\mathrm{H})=-\sum_{i=1}^{S} p i \ln p i$, Simpson's Index $(\lambda)=\sum_{i=1}^{S} p i^{2}$

Where, $p$ is the proportion $(\mathrm{n} / \mathrm{N})$ of individuals of one particular species found (n) divided by the total number of individuals found $(\mathrm{N})$, $\ln$ is the natural $\log , \Sigma$ is the sum of the calculations and $S$ is the number of species. Optimum values of $\mathrm{H}$ are generally between 1.5 and 3.5 in most ecological researches, and the index is rarely greater than 4. Shannon's Diversity Index is mathematical measurement to define community composition (number of species) and commonness of species in a community. It increases as both the richness and the evenness of the community increase (Shannon and Weiner 1949). Opposite to this, the Simpson's Index $(\lambda)$ is the mathematical representation of the similarity index. The range of $\lambda$ is from 0 to 1 (Simpson 1949). Higher the index means lower the diversity. It is the strength of dominance, because it weights towards the abundance of the most common species and varies inversely with species diversity (Whittaker 1972).

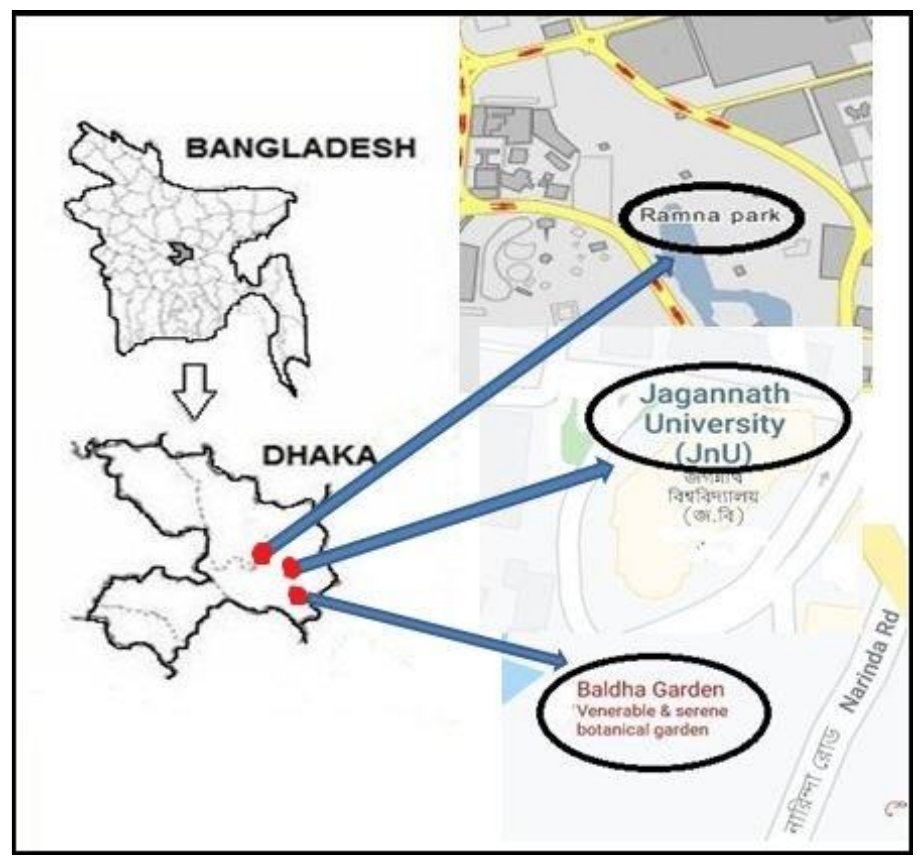

Fig. 1. Map of the Dhaka city in Bangladesh indicating the three study areas.

Simpson's Index of Diversity (1- 1$)$ : It is a measure of diversity. The probability of two randomly selected individuals in a community belongs to different categories e.g. species. The value of $1-\lambda$ ranges between 0 and 1 , where, high 
scores (close to 1) show high diversity and low scores (close to 0) show low diversity (Simpson 1949).

Simpson's Reciprocal Index (1/ג): The relative biodiversity of a community can be measured by it. It can be used to compare communities to identify intrinsic qualities. A high index value indicates a stable site with many different richness and low competition. A low index value shows a site with a few potential niches where only a few species dominate. The index value may alter in response to the ecological interference (MacDonald et al. 2017).

Species evenness (E): It refers to how close in number each species an environment is. The equation is: $\mathrm{E}=\frac{H}{\ln (S)}$. The range of $\mathrm{E}$ is from 0 to 1 . The value of $\mathrm{E}$ trends to 0 indicates that the species is more dominant in a community, $\mathrm{E}$ nears to 1 refers to evenly distributed (Shannon and Weiner 1949).

Community Similarity: It is measured by Sorenson's Coefficient (CC). The equation is: Sorenson's Coefficient $(\mathrm{CC})==\frac{3 \mathrm{C}}{\mathrm{S} 1+\mathrm{S} 2+\mathrm{S} 3}$, Where $\mathrm{C}$ is the number of species the three communities have in common, $S_{1}$ is the total number of species found in community $1, \mathrm{~S}_{2}$ is the total number of species found in community and $S_{3}$ is the total number of species found in community 3 (Sorensen 1948). Sorenson's coefficient gives a value between 0 and 1, the closer the value is to 1 , the more the communities have in common. Entire community overlap is equal to 1 ; entire community dissimilarity is equal to 0 (Sorensen 1948).

\section{RESULTS AND DISCUSSION}

A total of 8003 butterflies of 75 species belonging to 42 genera under 8 families were recorded from the Ramna Park, Jagannath University Campus and Baldha Garden in Dhaka city (Table 1, 2 and 3). Of them, 52 species (6253 individuals) under 8 families were found in the Ramna Park (Table 1), 37 species (1430 individuals) belongings to 7 families in the Jagannath University Campus (Table 2) and 20 species (320 individuals) of under 6 families in the Baldha Garden (Table 3). In these three study areas, 17 species, 14 genera and 6 families were in common.

About 430 species of butterfly are known to Bangladesh (Bashar 2014). Out of the above estimated species only 75 species of 8 families were recorded from the three selected areas in the Dhaka city in this study. Present investigation revealed that there were no new species, these areas were excellent for the occurrence of common species availability, Sarder et al. (2016) stated that in disturbed habitats and anthropogenic affected sites species richness was 
Table 1: Illustration how the various indices change as the relative number of each butterfly species change in the Ramna Park

\begin{tabular}{|c|c|c|c|c|c|c|c|c|c|c|c|c|c|}
\hline \multirow[t]{2}{*}{ Family name } & \multirow[t]{2}{*}{ Scientific name } & \multirow[t]{2}{*}{ Common name } & \multicolumn{6}{|c|}{ Monthly Abundance } & \multirow{2}{*}{$\begin{array}{c}\text { Total } \\
\text { (n) }\end{array}$} & \multirow[t]{2}{*}{ Status } & \multirow[t]{2}{*}{$\mathrm{Pi}$} & \multirow[t]{2}{*}{$P i^{2}$} & \multirow[t]{2}{*}{ PilnPi } \\
\hline & & & Jan & Feb & Mar & Apr & May & Jun & & & & & \\
\hline \multirow[t]{4}{*}{ Danaidae } & Danaus chrysippus & Plain Tiger & 12 & 14 & 18 & 27 & 24 & 31 & 126 & VC & 0.020150328 & 0.000406036 & -0.078677655 \\
\hline & Danaus genutia & Striped Tiger & 8 & 15 & 22 & 19 & 25 & 24 & 113 & vc & 0.018071326 & 0.000326573 & -0.072527979 \\
\hline & Tirumala limniace exoticus & Blue Tiger & 9 & 12 & 18 & 19 & 16 & 17 & 91 & c & 0.014553015 & 0.00021179 & -0.061558628 \\
\hline & Euploea core & Common Crow & 16 & 14 & 23 & 34 & 28 & 30 & 145 & vc & 0.023188869 & 0.000537724 & -0.087284826 \\
\hline \multirow[t]{8}{*}{ Papilionidae } & Pachliopta aristolochiae & Common Rose & 7 & 10 & 15 & 12 & 17 & 13 & 74 & c & 0.01183432 & 0.000140051 & -0.052505935 \\
\hline & Pachliopta hector & Crimson Rose & 1 & 0 & 3 & 2 & 5 & 1 & 12 & R & 0.001919079 & $3.68286 \mathrm{E}-06$ & -0.012005584 \\
\hline & Papilio polytes romulus & Common Mormon & 6 & 11 & 18 & 29 & 24 & 32 & 120 & vc & 0.019190788 & 0.000368286 & -0.075867421 \\
\hline & Papilio polymnestor & Blue Mormon & 2 & 9 & 14 & 11 & 8 & 13 & 57 & c & 0.009115625 & $8.30946 \mathrm{E}-05$ & -0.042823065 \\
\hline & Papilio demoleus & Lime Butterfly & 6 & 10 & 18 & 12 & 16 & 21 & 83 & c & 0.013273629 & 0.000176189 & -0.057368305 \\
\hline & Papilio clytia & Common Mime & 1 & 7 & 12 & 9 & 14 & 17 & 60 & c & 0.009595394 & $9.20716 \mathrm{E}-05$ & -0.044584731 \\
\hline & $\begin{array}{l}\text { Graphium agamemnon } \\
\text { Graphium doson axion }\end{array}$ & $\begin{array}{l}\text { Tailed Jay } \\
\text { Common Jay }\end{array}$ & $\begin{array}{l}0 \\
11\end{array}$ & $\begin{array}{l}2 \\
10\end{array}$ & $\begin{array}{l}6 \\
27\end{array}$ & $\begin{array}{l}5 \\
22\end{array}$ & $\begin{array}{l}3 \\
25\end{array}$ & $\begin{array}{l}8 \\
32\end{array}$ & $\begin{array}{l}24 \\
127\end{array}$ & $\begin{array}{l}\text { NR } \\
\text { VC }\end{array}$ & $\begin{array}{l}0.003838158 \\
0.020310251\end{array}$ & $\begin{array}{l}1.47315 E-05 \\
0.000412506\end{array}$ & $\begin{array}{l}-0.021350761 \\
-0.079141524\end{array}$ \\
\hline & Graphium sarpedon & $\begin{array}{l}\text { Common } \\
\text { Bluebottle }\end{array}$ & 2 & 4 & 1 & 6 & 8 & 5 & 26 & NR & 0.004158004 & 1.7289E-05 & -0.022797173 \\
\hline \multirow[t]{7}{*}{ Pieridae } & $\begin{array}{l}\text { Catopsilia pyranthe } \\
\text { Catopsilia pomona }\end{array}$ & $\begin{array}{l}\text { Mottled Emigrant } \\
\text { Lemon Emigrant }\end{array}$ & $\begin{array}{l}8 \\
16\end{array}$ & $\begin{array}{l}11 \\
31\end{array}$ & $\begin{array}{l}18 \\
38\end{array}$ & $\begin{array}{l}14 \\
25\end{array}$ & $\begin{array}{l}10 \\
42\end{array}$ & $\begin{array}{l}18 \\
36\end{array}$ & $\begin{array}{l}79 \\
188\end{array}$ & $\begin{array}{l}\mathrm{C} \\
\mathrm{VC}\end{array}$ & $\begin{array}{l}0.012633936 \\
0.030065569\end{array}$ & $\begin{array}{l}0.000159616 \\
0.000903938\end{array}$ & $\begin{array}{l}-0.055227592 \\
-0.105361017\end{array}$ \\
\hline & Eurema blanda silhetana & $\begin{array}{l}\text { Three-Spot Grass } \\
\text { Yellow }\end{array}$ & 84 & 90 & 121 & 133 & 98 & 112 & 638 & vc & 0.102031025 & 0.01041033 & -0.232883605 \\
\hline & Eurema hecabe hecabe & $\begin{array}{l}\text { Common Grass } \\
\text { Yellow }\end{array}$ & 38 & 44 & 32 & 42 & 128 & 61 & 345 & vc & 0.055173517 & 0.003044117 & -0.159852697 \\
\hline & Appias libythea & Striped Albatross & 18 & 13 & 22 & 31 & 38 & 29 & 151 & vc & 0.024148409 & 0.000583146 & -0.089917488 \\
\hline & $\begin{array}{l}\text { Leptosia nina } \\
\text { Delias eucharis }\end{array}$ & $\begin{array}{l}\text { Psyche } \\
\text { Common Jezebel }\end{array}$ & $\begin{array}{l}48 \\
12\end{array}$ & $\begin{array}{l}34 \\
19\end{array}$ & $\begin{array}{l}44 \\
28\end{array}$ & $\begin{array}{l}66 \\
46\end{array}$ & $\begin{array}{l}47 \\
34\end{array}$ & $\begin{array}{l}52 \\
31\end{array}$ & $\begin{array}{l}291 \\
170\end{array}$ & $\begin{array}{l}\text { vc } \\
\text { vc }\end{array}$ & $\begin{array}{l}0.046537662 \\
0.02718695\end{array}$ & $\begin{array}{l}0.002165754 \\
0.00073913\end{array}$ & $\begin{array}{l}-0.142753969 \\
-0.09800945\end{array}$ \\
\hline & Cepora nerissa & Common Gull & 0 & 4 & 12 & 7 & 9 & 8 & 40 & NR & 0.006396929 & 4.09207E-05 & -0.032316886 \\
\hline & Artogeia canidia indica & $\begin{array}{l}\text { Indian Cabbage } \\
\text { White }\end{array}$ & 5 & 3 & 16 & 18 & 8 & 6 & 56 & c & 0.008955701 & $8.02046 \mathrm{E}-05$ & -0.042230295 \\
\hline \multirow[t]{6}{*}{ Nymphalidae } & Phalanta phalantha & Common Leopard & 12 & 28 & 19 & 22 & 15 & 17 & 113 & vc & 0.018071326 & 0.000326573 & -0.072527979 \\
\hline & $\begin{array}{l}\text { Hypolimnas bolina bolina } \\
\text { Ariadne merione tapestrina }\end{array}$ & $\begin{array}{l}\text { Great Eggfly } \\
\text { Common Castor }\end{array}$ & $\begin{array}{l}23 \\
11\end{array}$ & $\begin{array}{l}26 \\
18\end{array}$ & $\begin{array}{l}28 \\
37\end{array}$ & $\begin{array}{l}33 \\
32\end{array}$ & $\begin{array}{l}21 \\
42\end{array}$ & $\begin{array}{l}16 \\
26\end{array}$ & $\begin{array}{l}147 \\
166\end{array}$ & $\begin{array}{l}\text { VC } \\
\text { VC }\end{array}$ & $\begin{array}{l}0.023508716 \\
0.026547257\end{array}$ & $\begin{array}{l}0.00055266 \\
0.000704757\end{array}$ & $\begin{array}{l}-0.088166713 \\
-0.096335453\end{array}$ \\
\hline & Euthalia aconthea & Common Baron & 18 & 14 & 32 & 44 & 47 & 33 & 188 & vc & 0.030065569 & 0.000903938 & -0.105361017 \\
\hline & Junonia atilites & Grey Pansy & 7 & 13 & 28 & 31 & 18 & 16 & 113 & vc & 0.018071326 & 0.000326573 & -0.072527979 \\
\hline & $\begin{array}{l}\text { Junonia almanac } \\
\text { Junonia onithya }\end{array}$ & $\begin{array}{l}\text { Peacock Pansy } \\
\text { Blue Pansy }\end{array}$ & $\begin{array}{l}19 \\
0\end{array}$ & $\begin{array}{l}10 \\
6\end{array}$ & $\begin{array}{l}29 \\
8\end{array}$ & $\begin{array}{l}33 \\
15\end{array}$ & $\begin{array}{l}42 \\
4\end{array}$ & $\begin{array}{l}14 \\
7\end{array}$ & $\begin{array}{l}147 \\
40\end{array}$ & $\begin{array}{l}\mathrm{VC} \\
\mathrm{NR}\end{array}$ & $\begin{array}{l}0.023508716 \\
0.006396929\end{array}$ & $\begin{array}{l}0.00055266 \\
4.09207 \mathrm{E}-05\end{array}$ & $\begin{array}{l}-0.088166713 \\
-0.032316886\end{array}$ \\
\hline & $\begin{array}{l}\text { Junonia lemonias } \\
\text { Neptis hylas }\end{array}$ & $\begin{array}{l}\text { Lemon Pansy } \\
\text { Common Sailor }\end{array}$ & $\begin{array}{l}16 \\
28\end{array}$ & $\begin{array}{l}27 \\
31\end{array}$ & $\begin{array}{l}34 \\
39\end{array}$ & $\begin{array}{l}28 \\
31\end{array}$ & $\begin{array}{l}46 \\
37\end{array}$ & $\begin{array}{l}31 \\
21\end{array}$ & $\begin{array}{l}182 \\
187\end{array}$ & $\begin{array}{l}\text { vc } \\
\text { vc }\end{array}$ & $\begin{array}{l}0.029106029 \\
0.029905645\end{array}$ & $\begin{array}{l}0.000847161 \\
0.000894348\end{array}$ & $\begin{array}{l}-0.102942493 \\
-0.104960083\end{array}$ \\
\hline \multirow[t]{11}{*}{ Lycaenidae } & Castalius rosimon & Common Pierrot & 7 & 16 & 21 & 31 & 27 & 23 & 125 & vc & 0.019990405 & 0.000399616 & -0.078212516 \\
\hline & Jamides bochus & Dark Cerulean & 3 & 18 & 23 & 16 & 27 & 6 & 93 & c & 0.014872861 & 0.000221202 & -0.062588229 \\
\hline & Zizeeria karsandra & Dark Grass Blue & 18 & 23 & 31 & 39 & 12 & 15 & 138 & vc & 0.022069407 & 0.000487059 & -0.084163071 \\
\hline & Pseudozizeeria maha maha & Pale Grass Blue & 25 & 33 & 43 & 17 & 21 & 9 & 148 & vc & 0.023668639 & 0.000560204 & -0.08860602 \\
\hline & Prosotas dubiosa & Tailless Line Blue & 1 & 8 & 2 & 0 & 3 & 0 & 14 & $\mathrm{R}$ & 0.002238925 & $5.01279 E-06$ & -0.013661383 \\
\hline & Neopithecops zalmora & Quaker & 6 & 11 & 9 & 4 & 15 & 7 & 52 & c & 0.008316008 & $6.9156 \mathrm{E}-05$ & -0.039830128 \\
\hline & Euchrysops cnejus & Gram Blue & 18 & 16 & 31 & 17 & 13 & 11 & 106 & vc & 0.016951863 & 0.000287366 & -0.069119146 \\
\hline & Chilades lajus & Lime Blue & 11 & 17 & 12 & 14 & 21 & 8 & 83 & c & 0.013273629 & 0.000176189 & -0.057368305 \\
\hline & Chilades pandava & Plains Cupid & 0 & 2 & 7 & 3 & 2 & 1 & 15 & NR & 0.002398849 & $5.75447 \mathrm{E}-06$ & -0.014471693 \\
\hline & Lampides boeticus & Pea Blue & 28 & 31 & 34 & 43 & 39 & 33 & 208 & vc & 0.033264033 & 0.001106496 & -0.113206771 \\
\hline & Catochrysops strabo & Forget-me-not & 17 & 21 & 18 & 27 & 32 & 15 & 130 & vc & 0.020790021 & 0.000432225 & -0.080525617 \\
\hline \multirow[t]{7}{*}{ Hesperidae } & Saustrus gremius & Indian Palm Bob & 9 & 7 & 0 & 6 & 4 & 3 & 29 & NR & 0.004637774 & 2.15089E-05 & -0.024921174 \\
\hline & Borbo cinnara & Rice Swift & 6 & 11 & 21 & 17 & 14 & 15 & 84 & C & 0.013433552 & 0.00018046 & -0.057898606 \\
\hline & Pelopidas conjuncta & Conjoined Swift & 15 & 21 & 27 & 12 & 3 & 11 & 89 & c & 0.014233168 & 0.000202583 & -0.060521996 \\
\hline & Pamara guttatus mangala & Straight Swift & 0 & 2 & 7 & 4 & 19 & 33 & 65 & c & 0.01039501 & 0.000108056 & -0.047468081 \\
\hline & Oriens gola & Common Dartlet & 0 & 3 & 1 & 7 & 3 & 2 & 16 & NR & 0.002558772 & $6.54731 \mathrm{E}-06$ & -0.015271333 \\
\hline & Oriens goloides & Smaller Dartlet & 3 & 11 & 15 & 12 & 19 & 17 & 77 & c & 0.012314089 & 0.000151637 & -0.054145188 \\
\hline & Badamia exclamationis & Brown Awl & & & 3 & & 0 & & & NR & 0.003038542 & $9.23273 E-06$ & -0.017612534 \\
\hline \multirow[t]{2}{*}{ Satyridae } & Elymnias hypermnestra & Common Palmfly & 8 & 14 & 21 & 18 & 25 & 23 & 109 & vc & 0.017431633 & 0.000303862 & -0.070588852 \\
\hline & Melanitis leda & $\begin{array}{l}\text { Common Evening } \\
\text { Brown }\end{array}$ & 18 & 21 & 27 & 31 & 46 & 37 & 180 & vc & 0.028786183 & 0.000828644 & -0.102129339 \\
\hline Acriidae & Acraea violae & TwanyCoster & 23 & 35 & 29 & 24 & 41 & 22 & 174 & vc & 0.027826643 & 0.000774322 & -0.099668395 \\
\hline & $S=52$ & & 662 & 866 & 1162 & 1202 & 1285 & 1076 & 6253 & & & 0.032403906 & -3.682330281 \\
\hline
\end{tabular}

The maximum population was recorded in the month of May $(20.55 \%)$ followed by April (19.22\%), March (18.58\%), June (17.21\%), February (13.85\%) and the minimum of January (10.59\%) (Fig. 6). increased but the uniqueness was less. These results are in good agreement with Padhey et al. (2012).

At the Ramna Park, family-wise species richness and butterfly population are shown in Fig. 3 and 5, respectively. The maximum species richness included the family Lycaenidae $(21.15 \%, 11$ spp.) followed by Papilionidae $(17.31 \%, 9$ 
Table 2. Illustration how the various indices change as the relative number of each butterfly species change in the Jagannath University Campus

\begin{tabular}{|c|c|c|c|c|c|c|c|c|c|c|c|c|c|}
\hline \multirow{2}{*}{$\begin{array}{l}\text { Family } \\
\text { Name }\end{array}$} & \multirow[t]{2}{*}{ Scientific name } & \multirow[t]{2}{*}{ Common name } & \multicolumn{6}{|c|}{ Monthly Abundance } & \multirow[t]{2}{*}{ Total } & \multirow[t]{2}{*}{ Status } & \multirow[t]{2}{*}{$\mathrm{Pi}$} & \multirow[t]{2}{*}{$P i^{2}$} & \multirow[t]{2}{*}{ PilnPi } \\
\hline & & & Jan & Feb & Mar & Apr & May & Jun & & & & & \\
\hline \multirow[t]{4}{*}{ Danaidae } & $\begin{array}{l}\text { Danaus } \\
\text { chrysippus }\end{array}$ & Plain Tiger & 11 & 15 & 21 & 25 & 14 & 0 & 86 & C & 0.06013986 & 0.003616803 & -0.169058104 \\
\hline & Danaus genutia & Striped Tiger & 8 & 12 & 9 & 2 & 0 & 0 & 31 & NR & 0.021678322 & 0.00046995 & -0.083059243 \\
\hline & $\begin{array}{l}\text { Tirumala limniace } \\
\text { exoticus }\end{array}$ & Blue Tiger & 2 & 1 & 7 & 5 & 1 & 0 & 16 & NR & 0.011188811 & 0.000125189 & -0.05026955 \\
\hline & Euploea core & Common Crow & 1 & 4 & 13 & 5 & 1 & 0 & 24 & NR & 0.016783217 & 0.000281676 & -0.068599316 \\
\hline \multirow[t]{5}{*}{ Papilionidae } & $\begin{array}{l}\text { Pachliopta } \\
\text { aristolochiae }\end{array}$ & Common Rose & 12 & 10 & 2 & 1 & 0 & 0 & 25 & NR & 0.017482517 & 0.000305638 & -0.070743949 \\
\hline & $\begin{array}{l}\text { Papilio polytes } \\
\text { romulus }\end{array}$ & $\begin{array}{l}\text { Common } \\
\text { Mormon }\end{array}$ & 6 & 11 & 14 & 17 & 12 & 11 & 71 & C & 0.04965035 & 0.002465157 & -0.14908758 \\
\hline & Papilio demoleus & Lime Butterfly & 6 & 10 & 11 & 8 & 13 & 2 & 50 & C & 0.034965035 & 0.001222554 & -0.117251983 \\
\hline & Papilio clytia & Common Mime & 1 & 3 & 0 & 0 & 1 & 2 & 7 & $\mathrm{R}$ & 0.004895105 & 2.39621E-05 & -0.026039606 \\
\hline & $\begin{array}{l}\text { Graphium doson } \\
\text { axion }\end{array}$ & Common Jay & 0 & 0 & 2 & 7 & 4 & 3 & 16 & NR & 0.011188811 & 0.000125189 & -0.05026955 \\
\hline \multirow[t]{7}{*}{ Pieridae } & $\begin{array}{l}\text { Catopsilia } \\
\text { pyranthe }\end{array}$ & Mottled Emigrant & 1 & 5 & 9 & 2 & 0 & 1 & 18 & NR & 0.012587413 & 0.000158443 & -0.05507066 \\
\hline & $\begin{array}{l}\text { Catopsilia } \\
\text { pomona }\end{array}$ & Lemon Emigrant & 0 & 3 & 8 & 15 & 12 & 6 & 44 & NR & 0.030769231 & 0.000946746 & -0.10711508 \\
\hline & $\begin{array}{l}\text { Eurema blanda } \\
\text { silhetana }\end{array}$ & $\begin{array}{l}\text { Three-Spot } \\
\text { Grass Yellow }\end{array}$ & 28 & 35 & 44 & 42 & 31 & 33 & 213 & VC & 0.148951049 & 0.022186415 & -0.283623287 \\
\hline & $\begin{array}{l}\text { Eurema hecabe } \\
\text { hecabe }\end{array}$ & $\begin{array}{l}\text { Common Grass } \\
\text { Yellow }\end{array}$ & 18 & 24 & 23 & 31 & 28 & 27 & 151 & VC & 0.105594406 & 0.011150178 & -0.237392051 \\
\hline & Appias libythea & Striped Albatross & 11 & 13 & 0 & 0 & 1 & 2 & 27 & NR & 0.018881119 & 0.000356497 & -0.074950355 \\
\hline & Leptosia nina & Psyche & 8 & 14 & 18 & 1 & 0 & 0 & 41 & NR & 0.028671329 & 0.000822045 & -0.101836478 \\
\hline & Delias eucharis & $\begin{array}{l}\text { Common } \\
\text { Jezebel }\end{array}$ & 2 & 0 & 0 & 0 & 1 & 0 & 3 & $\mathrm{R}$ & 0.002097902 & 4.40119E-06 & -0.012937379 \\
\hline \multirow[t]{4}{*}{ Nymphalidae } & $\begin{array}{l}\text { Phalanta } \\
\text { phalantha }\end{array}$ & $\begin{array}{l}\text { Common } \\
\text { Leopard }\end{array}$ & 12 & 28 & 19 & 22 & 15 & 17 & 113 & VC & 0.079020979 & 0.006244315 & -0.200558556 \\
\hline & $\begin{array}{l}\text { Hypolimnas } \\
\text { bolina bolina }\end{array}$ & Great Eggfly & 5 & 1 & 14 & 11 & 8 & 3 & 42 & NR & 0.029370629 & 0.000862634 & -0.103612535 \\
\hline & $\begin{array}{l}\text { Junonia atlites } \\
\text { atlites }\end{array}$ & Grey Pansy & 4 & 3 & 12 & 7 & 2 & 2 & 30 & NR & 0.020979021 & 0.000440119 & -0.081067811 \\
\hline & Junonia almanac & Peacock Pansy & 1 & 0 & 4 & 7 & 10 & 1 & 23 & NR & 0.016083916 & 0.000258692 & -0.066425536 \\
\hline \multirow[t]{8}{*}{ Lycaenidae } & Castalius rosimon & Common Pierrot & 9 & 7 & 15 & 13 & 5 & 3 & 52 & C & 0.036363636 & 0.001322314 & -0.120515855 \\
\hline & Jamides bochus & Dark Cerulean & 3 & 1 & 7 & 10 & 0 & 0 & 21 & NR & 0.014685315 & 0.000215658 & -0.061985352 \\
\hline & $\begin{array}{l}\text { Zizeeria } \\
\text { karsandra }\end{array}$ & Dark Grass Blue & 0 & 2 & 5 & 9 & 7 & 9 & 32 & NR & 0.022377622 & 0.000500758 & -0.085028113 \\
\hline & $\begin{array}{l}\text { Pseudozizeeria } \\
\text { maha }\end{array}$ & Pale Grass Blue & 5 & 13 & 11 & 6 & 8 & 7 & 50 & C & 0.034965035 & 0.001222554 & -0.117251983 \\
\hline & Prosotas dubiosa & $\begin{array}{l}\text { Tailless Line } \\
\text { Blue }\end{array}$ & 0 & 3 & 5 & 9 & 12 & 0 & 29 & NR & 0.02027972 & 0.000411267 & -0.079053065 \\
\hline & $\begin{array}{l}\text { Euchrysops } \\
\text { cnejus }\end{array}$ & Gram Blue & 5 & 7 & 11 & 6 & 3 & 2 & 34 & NR & 0.023776224 & 0.000565309 & -0.088900946 \\
\hline & Chilades lajus & Lime Blue & 3 & 1 & 8 & 10 & 5 & 4 & 31 & NR & 0.021678322 & 0.00046995 & -0.083059243 \\
\hline & $\begin{array}{l}\text { Lampides } \\
\text { boeticus }\end{array}$ & Pea Blue & 0 & 4 & 3 & 1 & 0 & 0 & 8 & $\mathrm{R}$ & 0.005594406 & $3.12974 \mathrm{E}-05$ & -0.029012521 \\
\hline \multirow[t]{8}{*}{ Hesperiidae } & Saustrus gremius & Indian Palm Bob & 7 & 5 & 3 & 4 & 0 & 0 & 19 & NR & 0.013286713 & 0.000176537 & -0.057411765 \\
\hline & Borbo cinnara & Rice Switt & 3 & 2 & 0 & 5 & 4 & 4 & 18 & NR & 0.012587413 & 0.000158443 & -0.05507066 \\
\hline & $\begin{array}{l}\text { Pelopidas } \\
\text { conjuncta }\end{array}$ & Conjoined Swift & 3 & 2 & 3 & 0 & 0 & 0 & 8 & $\mathrm{R}$ & 0.005594406 & $3.12974 \mathrm{E}-05$ & -0.029012521 \\
\hline & $\begin{array}{l}\text { Pamara guttatus } \\
\text { mangala }\end{array}$ & Straight Swift & 2 & 4 & 2 & 1 & 2 & 0 & 11 & $R$ & 0.007692308 & $5.91716 \mathrm{E}-05$ & -0.037442573 \\
\hline & Tagiade sjapetus & Pied Flat & 0 & 1 & 2 & 0 & 0 & 0 & 3 & $R$ & 0.002097902 & 4.40119E-06 & -0.012937379 \\
\hline & Oriens gola & Common Dartlet & 1 & 3 & 3 & 2 & 0 & 0 & 9 & $R$ & 0.006293706 & 3.96107E-05 & -0.031897795 \\
\hline & Oriens goloides & Smaller Dartlet & 3 & 2 & 3 & 0 & 1 & 0 & 9 & $\mathrm{R}$ & 0.006293706 & 3.96107E-05 & -0.031897795 \\
\hline & $\begin{array}{l}\text { Badamia } \\
\text { exclamationis }\end{array}$ & Brown Awl & 0 & 4 & 7 & 15 & 13 & 5 & 44 & NR & 0.030769231 & 0.000946746 & -0.10711508 \\
\hline Satyridae & $\begin{array}{l}\text { Mycalesis } \\
\text { perseus blasius }\end{array}$ & $\begin{array}{l}\text { Common } \\
\text { Bushbrown }\end{array}$ & 3 & 8 & 7 & 2 & 0 & 1 & 21 & NR & 0.014685315 & 0.000215658 & -0.061985352 \\
\hline 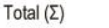 & $S=37$ & & 184 & 261 & 325 & 301 & 214 & 145 & 1430 & & 1 & 0.058477187 & -3.198546606 \\
\hline
\end{tabular}

spp.), Pieridae (17.31\%, 9 spp.), Nymphalidae (17.31\%, 9 spp.), Hesperiidae (13.46\%, 7 spp.), Danaidae (7.69\%, 4 spp.), Satyridae (3.85\%, 2 spp.), and the minimum was in the family Acriidae (1.92\%, 1 spp.). The highest butterfly 
population was in the family Pieridae (1958 individuals) followed by Nymphalidae (1283 individuals), Lycaenidae (1112 individuals), Papilionidae (583 individuals), Danaidae ( 475 individuals), Hesperiidae (379 individuals), Satyridae (289 individuals), and the lowest was in the family Acriidae (174 individuals) (Fig. 5).Among them, Eurema blanda silhetana $(\mathrm{n}=638)$ was the most dominant species followed by Eurema hecabe $(\mathrm{n}=345)$ and Leptosia nina $(\mathrm{n}=291)$ (Table 1). On the other hand, Pachliopta hector $(\mathrm{n}=12)$ was in the lowest population followed by Prosotas dubiosa $(\mathrm{n}=14)$ Chilades pandava $(\mathrm{n}=15)$, Oriens gola $(\mathrm{n}=16)$ and

Table 3. Illustration how the various indices change as the relative number of each butterfly species change in the Baldha Garden

\begin{tabular}{|c|c|c|c|c|c|c|c|c|c|c|c|c|c|}
\hline \multirow[t]{2}{*}{ Family name } & \multirow{2}{*}{$\begin{array}{c}\text { Scientific } \\
\text { name }\end{array}$} & \multirow{2}{*}{$\begin{array}{c}\text { Common } \\
\text { name }\end{array}$} & \multicolumn{6}{|c|}{ Monthly Abundance } & \multirow[t]{2}{*}{ Total } & \multirow[t]{2}{*}{ Status } & \multirow[t]{2}{*}{$\mathbf{P i}$} & \multirow[t]{2}{*}{$P i^{2}$} & \multirow[t]{2}{*}{ PilnPi } \\
\hline & & & Jan & Feb & Mar & Apr & May & Jun & & & & & \\
\hline \multirow[t]{2}{*}{ Danaidae } & $\begin{array}{l}\text { Danaus } \\
\text { chrysippus }\end{array}$ & Plain Tiger & 0 & 2 & 4 & 4 & 12 & 4 & 26 & $\mathrm{NR}$ & 0.08125 & 0.006601563 & -0.203955737 \\
\hline & Euploea core & $\begin{array}{l}\text { Common } \\
\text { Crow }\end{array}$ & 1 & 3 & 0 & 0 & 1 & 0 & 5 & $\mathrm{R}$ & 0.015625 & 0.000244141 & -0.064982548 \\
\hline \multirow[t]{3}{*}{ Papilionidae } & $\begin{array}{l}\text { Pachliopta } \\
\text { aristolochiae }\end{array}$ & $\begin{array}{l}\text { Common } \\
\text { Rose }\end{array}$ & 0 & 0 & 2 & 3 & 0 & 2 & 7 & $\mathrm{R}$ & 0.021875 & 0.000478516 & -0.083615237 \\
\hline & $\begin{array}{l}\text { Papilio } \\
\text { polytes } \\
\text { romulus }\end{array}$ & $\begin{array}{l}\text { Common } \\
\text { Mormon }\end{array}$ & 0 & 2 & 1 & 1 & 0 & 2 & 6 & $\mathrm{R}$ & 0.01875 & 0.000351563 & -0.074560529 \\
\hline & $\begin{array}{l}\text { Papilio } \\
\text { demoleus }\end{array}$ & $\begin{array}{l}\text { Lime } \\
\text { Butterfly }\end{array}$ & 0 & 0 & 0 & 0 & 2 & 0 & 2 & VR & 0.00625 & $3.90625 \mathrm{E}-05$ & -0.031719836 \\
\hline \multirow[t]{6}{*}{ Pieridae } & $\begin{array}{l}\text { Catopsilia } \\
\text { pomona }\end{array}$ & $\begin{array}{l}\text { Lemon } \\
\text { Emigrant }\end{array}$ & 0 & 3 & 2 & 7 & 4 & 3 & 19 & NR & 0.059375 & 0.003525391 & -0.167667995 \\
\hline & $\begin{array}{l}\text { Eurema } \\
\text { blanda } \\
\text { silhetana }\end{array}$ & $\begin{array}{l}\text { Three-Spot } \\
\text { Grass } \\
\text { Yellow }\end{array}$ & 0 & 5 & 3 & 10 & 12 & 9 & 39 & NR & 0.121875 & 0.014853516 & -0.256517546 \\
\hline & $\begin{array}{l}\text { Eurema } \\
\text { hecabe } \\
\text { hecabe }\end{array}$ & $\begin{array}{l}\text { Common } \\
\text { Grass } \\
\text { Yellow }\end{array}$ & 1 & 4 & 3 & 7 & 9 & 4 & 28 & NR & 0.0875 & 0.00765625 & -0.213160192 \\
\hline & $\begin{array}{l}\text { Lepto } \\
\text { sianina }\end{array}$ & Psyche & 5 & 4 & 4 & 6 & 0 & 1 & 20 & NR & 0.0625 & 0.00390625 & -0.173286795 \\
\hline & $\begin{array}{l}\text { Delias } \\
\text { eucharis }\end{array}$ & $\begin{array}{l}\text { Common } \\
\text { Jezebel }\end{array}$ & 2 & 0 & 0 & 0 & 1 & 0 & 3 & $\mathrm{R}$ & 0.009375 & $8.78906 \mathrm{E}-05$ & -0.043778519 \\
\hline & $\begin{array}{l}\text { Hypolimnas } \\
\text { bolinabolina }\end{array}$ & $\begin{array}{l}\text { Great } \\
\text { Eggfly }\end{array}$ & 1 & 2 & 0 & 3 & 1 & 0 & 7 & $\mathrm{R}$ & 0.021875 & 0.000478516 & -0.083615237 \\
\hline \multirow[t]{3}{*}{ Nymphalidae } & $\begin{array}{l}\text { Junonia } \\
\text { atlites atlites }\end{array}$ & $\begin{array}{l}\text { Grey } \\
\text { Pansy }\end{array}$ & 1 & 2 & 1 & 3 & 2 & 4 & 13 & $\mathrm{R}$ & 0.040625 & 0.001650391 & -0.130136973 \\
\hline & $\begin{array}{l}\text { Junonia } \\
\text { almanac }\end{array}$ & $\begin{array}{l}\text { Peacock } \\
\text { Pansy }\end{array}$ & 0 & 0 & 0 & 3 & 2 & 3 & 8 & $\mathrm{R}$ & 0.025 & 0.000625 & -0.092221986 \\
\hline & Neptishylas & $\begin{array}{l}\text { Common } \\
\text { Sailor }\end{array}$ & 2 & 0 & 1 & 3 & 4 & 0 & 10 & $\mathrm{R}$ & 0.03125 & 0.000976563 & -0.108304247 \\
\hline \multirow[t]{4}{*}{ Lycaenidae } & $\begin{array}{l}\text { Zizeeria } \\
\text { karsandra }\end{array}$ & $\begin{array}{l}\text { Dark } \\
\text { Grass } \\
\text { Blue }\end{array}$ & 0 & 2 & 1 & 3 & 3 & 0 & 9 & $\mathrm{R}$ & 0.028125 & 0.000791016 & -0.100437087 \\
\hline & $\begin{array}{l}\text { Pseudozizeer } \\
\text { iamaha }\end{array}$ & $\begin{array}{l}\text { Pale Grass } \\
\text { Blue }\end{array}$ & 8 & 11 & 15 & 18 & 15 & 24 & 91 & C & 0.284375 & 0.080869141 & -0.357590611 \\
\hline & Zizula hylax & $\begin{array}{l}\text { Tiny Grass } \\
\text { Blue }\end{array}$ & 0 & 0 & 3 & 1 & 1 & 0 & 5 & $\mathrm{R}$ & 0.015625 & 0.000244141 & -0.064982548 \\
\hline & $\begin{array}{l}\text { Euchrysops } \\
\text { cnejus }\end{array}$ & Gram Blue & 0 & 3 & 5 & 1 & 0 & 2 & 11 & $\mathrm{R}$ & 0.034375 & 0.001181641 & -0.115858384 \\
\hline \multirow[t]{2}{*}{ Hesperiidae } & $\begin{array}{l}\text { Parnara } \\
\text { guttatus } \\
\text { mangala }\end{array}$ & $\begin{array}{l}\text { Straight } \\
\text { Swift }\end{array}$ & 0 & 3 & 2 & 1 & 2 & 0 & 8 & $\mathrm{R}$ & 0.025 & 0.000625 & -0.092221986 \\
\hline & $\begin{array}{l}\text { Tagiades } \\
\text { japetus }\end{array}$ & Pied Flat & 0 & 1 & 2 & 0 & 0 & 0 & 3 & $\mathrm{R}$ & 0.009375 & $8.78906 \mathrm{E}-05$ & -0.043778519 \\
\hline 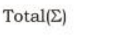 & $S=20$ & & 21 & 47 & 49 & 74 & 71 & 58 & 320 & & 1 & 0.125273438 & 2.502392514 \\
\hline
\end{tabular}

VC-Very Common (> 100 sightings), C - Common (50-99 sightings), NR - Not Rare (15-49 sightings), R - Rare (3-14 sightings), VR Very Rare (1-2 sightings) (Nidup et al. 2014).

Badamia exclamationis ( $\mathrm{n}=19)$ (Table 1). Among observed species, 28 were very common, 14 were common, 8 were not rare, and 2 were rare (Table 1). An analogous study was carried out by Koirala et al. (2020) at Gidakom Forest 
management Unit, Thimpu, Bhutan that revealed a total of 90 species under 52 genera and 5 families. Nymphalidae was dominant with 38 species followed by Lycaenidae with 19, Pieridae with 15, Papilionidae with 11, and Hesperiidae with 7 species.

Family-wise species richness and butterfly population are shown in figure 3 and 5, respectively at the Jagannath University Campus. The maximum species were recorded in the family Lycaenidae $(21.62 \%, 8$ spp.) and Hesperiidae (21.62\%, 8 spp.) followed by Pieridae (18.92\%, 7 spp.), Papilionidae (13.51\%, 5 spp.), Nymphalidae $(10.81 \%, 4$ spp.), Danaidae $(10.81 \%, 4$ spp.), and the minimum was in Satyridae $(2.70 \%, 1 \mathrm{spp}$.$) . The highest butterfly population was$ in the family Pieridae (497 individuals) followed by Lycaenidae (257 individuals), Nymphalidae (208 individuals), Papilionidae ( 169 individuals),

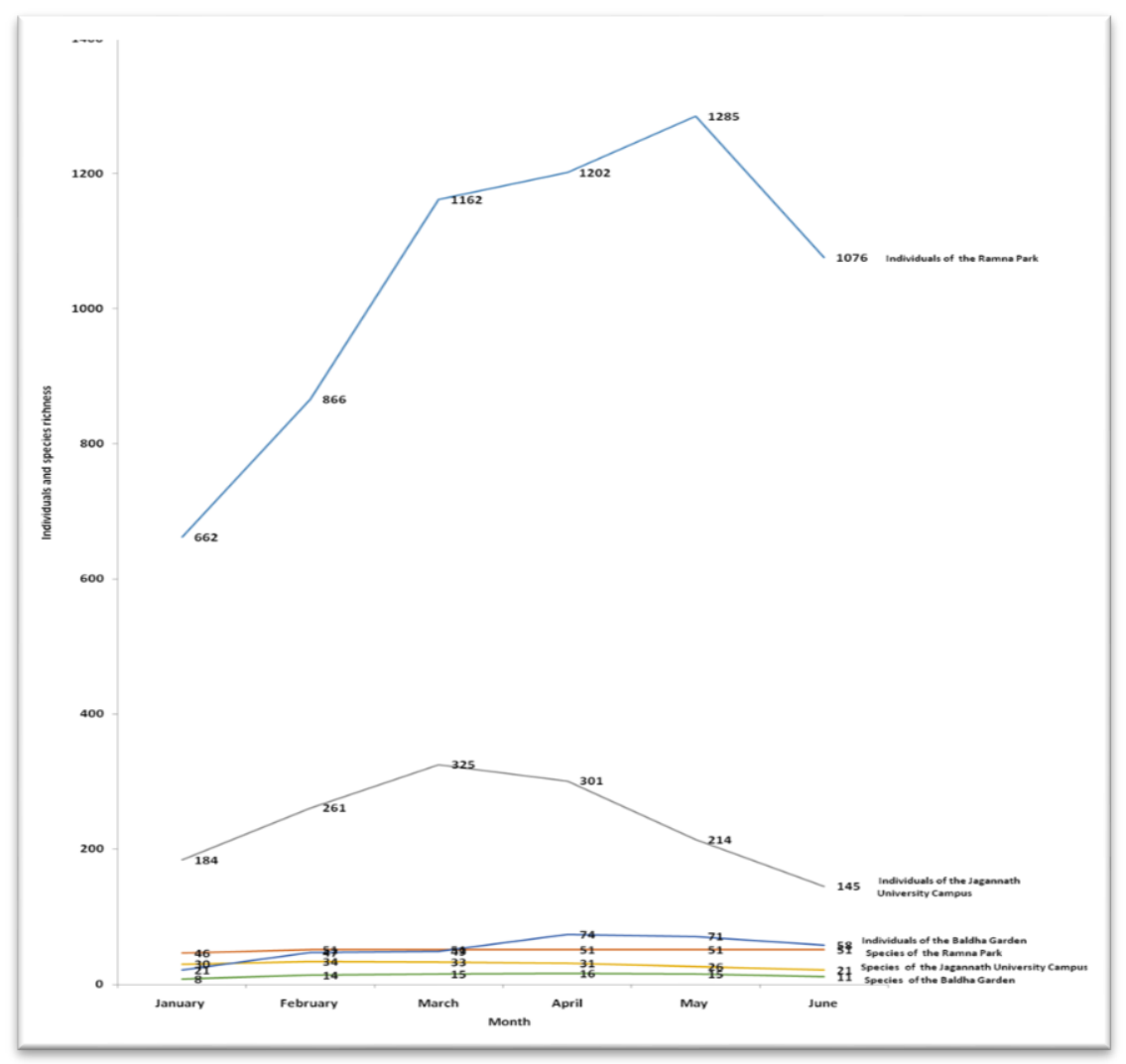

Fig. 2. Species richness and individuals curves for pooled data over six months.

Danaidae (157 individuals), Hesperiidae (121 individuals), and the lowest was in the family Satyridae (21 individuals). The maximum population was recorded in 
the month of March (22.73\%) followed by April (21.05\%), February (18.25\%), May (14.97\%), January (12.86\%) and the minimum of June (10.14\%) (Fig. 6). Among them, Eurema blanda silhetana $(n=213)$ was the most dominant species followed by Eurema hecabe $(\mathrm{n}=151)$ and Phalanta phalantha $(\mathrm{n}=113)$. On the other hand, Tagiades japetus $(\mathrm{n}=3)$ and Delias eucharis $(\mathrm{n}=3)$ were in the lowest

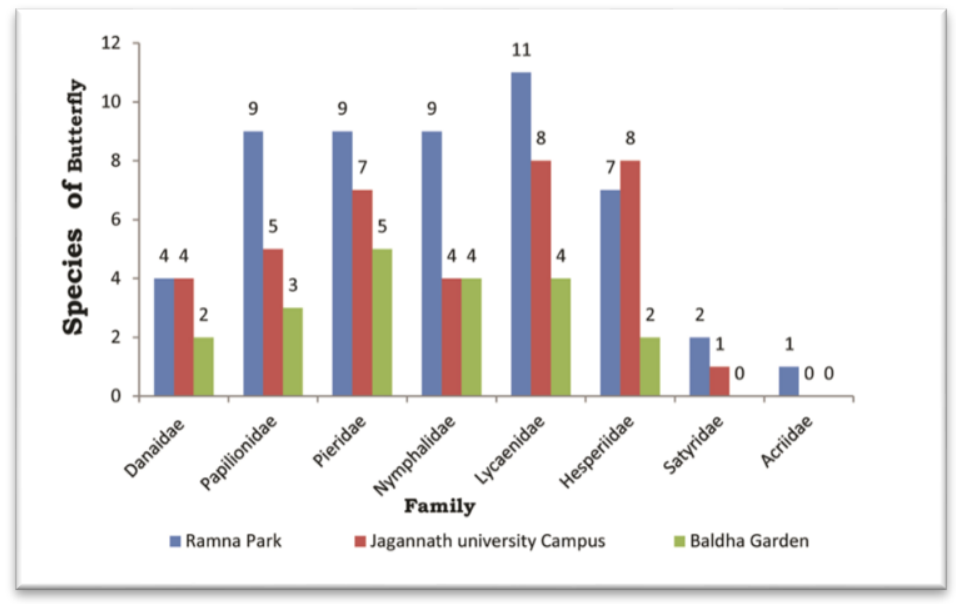

Fig. 3. Family-wise species at the three selected areas of the Dhaka city.

population followed by Papilio claytia $(\mathrm{n}=7)$, Lampides boeticus $(\mathrm{n}=8)$, Pelopidas conjuncta $(\mathrm{n}=8)$, Oriens gola $(\mathrm{n}=9)$, Oriens goloides $(\mathrm{n}=9)$, Parnara guttatus mangala $(\mathrm{n}=11)$, Graphium doson $(\mathrm{n}=16)$, Trirumala limniace $(\mathrm{n}=16)$, Catopsilia pyranthe $(\mathrm{n}=18)$, Borbo cinnara $(\mathrm{n}=18)$ and Saustrus gremius $(\mathrm{n}=19)$ (Table 2). Among observed species, 3 were very common, 5 were common, 21 were not rare and 8 were rare (Table 2). Similar work of Sarder et al. (2016) recorded 42 species under 8 families at Keranigonj, out of the recorded families, Pieridae $(36.59 \%)$ was in the highest population, then Nymphalidae $(24.08 \%)$, Lycaenidae (16.49\%), Dainaidae (13.89\%), Hesperiidae (6.45\%), Acriidae(1.29\%), Satyridae $(0.73 \%)$ and Papilionidae $(0.45 \%)$.

At the Baldha garden, family-wise species richness and butterfly population are shown in Fig. 3 and 5, respectively. The topmost species were recorded in the family Pieridae $(25 \%, 5$ spp.) followed by Lycaenidae $(20 \%, 4$ spp.), Nymphalidae (20\%, 4 spp.), Papilionidae (15\%, 3 spp.), and the lowest from the family Hesperiidae $(10 \%, 2$ spp.) and Danaidae $(10 \%, 2$ spp.) at the Baldha Garden. The highest butterfly population was in the family Lycaenidae (116 individuals) followed by Pieridae (109 individuals), Nymphalidae (38 
individuals), Danaidae (31 individuals), Papilionidae (15 individuals), and the lowest was in the family Hesperiidae (11 individuals). The maximum population was recorded in the month of April (23.12\%) followed by May (22.19\%), June (18.12\%), March (15.31\%), February (14.69\%) and the minimum of January (6.56\%) (Fig.6). Among them, Pseudozizeeria maha $(\mathrm{n}=91)$ was the most dominant species followed by Eurema blanda silhetana (n=39), Eurema hecabe $(\mathrm{n}=28)$ and Danaus crysippus $(\mathrm{n}=26)$. On the other hand, Papilio demoleus $(\mathrm{n}=2)$ was in the lowest population followed by Delias eucharis $(\mathrm{n}=3)$ Tagiades japetus $(\mathrm{n}=3)$, Euploea core $(\mathrm{n}=5)$, Zizula hylax $(\mathrm{n}=5)$ and Papilio polytes $(\mathrm{n}=6)$ (Table 3). Among observed species, 1 was common, 5 were not rare, 13 were rare, and 2 were very rare (Table 3). Nidup et al. (2014) recorded 91 species belonging to 5 major families of which 1 species was new in Royal Monas National Park, Bhutan. Nymphalidae was the most common $(33 \%, n=30)$, and the lowest was hesperiidae $(12 \%, \mathrm{n}=11)$.

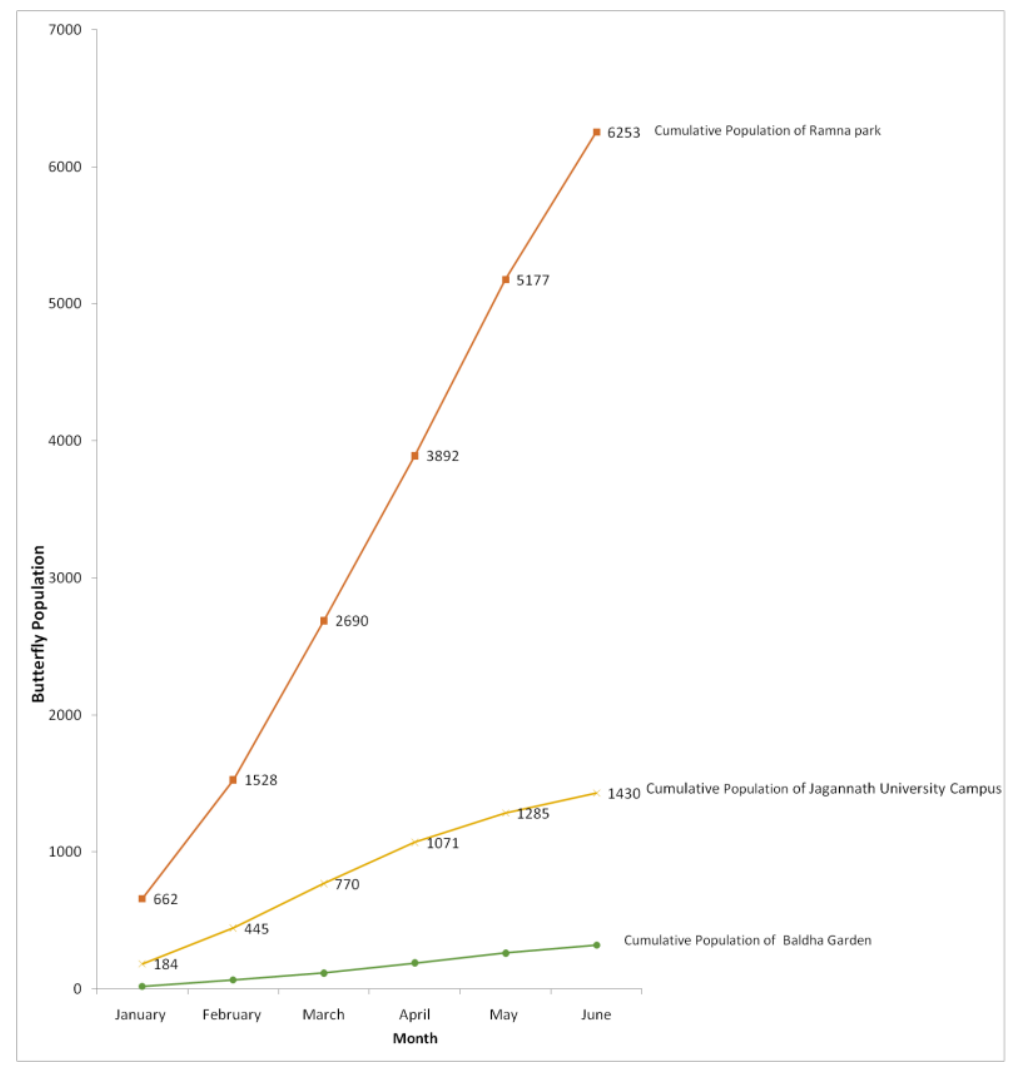

Fig. 4. Month-wise population cumulative curve of faunal assemblage of butterfly at the three selected areas of the Dhaka city. 
The value of various indices of species diversity in the Ramna Park, Jagannath University Campus and Baldha Garden are shown in Table 1, 2 and 3, respectively. The values of Shannon's Diversity Index $(\mathrm{H})$, the Simpson's Index $(\lambda)$, Simpson's Index of Diversity (1- $\lambda)$, Simpson's Reciprocal Index $(1 / \lambda)$ and the Species Evenness (E), are 3.68, 0.032, 0.97, 30.86 and 0.93, respectively for the Ramna Park (Table 1). The values of Shannon's Diversity Index $(H)$, the Simpson's Diversity Index ( $\lambda)$, Simpson's Index of Diversity (1- $\lambda)$, Simpson's Reciprocal Index $(1 / \lambda)$ and the Species Evenness (E) are 3.20, 0.058, .094, 17.1 and 0.89 , respectively for the Jagannath University (Table 2). The values of Shannon's Diversity Index $(H)$, the Simpson's Index $(\lambda)$, Simpson Index of Diversity (1- $\lambda)$ and Simpson Reciprocal Index $(1 / \lambda)$ and the Species Evenness (E) are 2.50, 0.13, 0.87, 7.98 and 0.84, respectively for the Baldha Garden (Table 3).

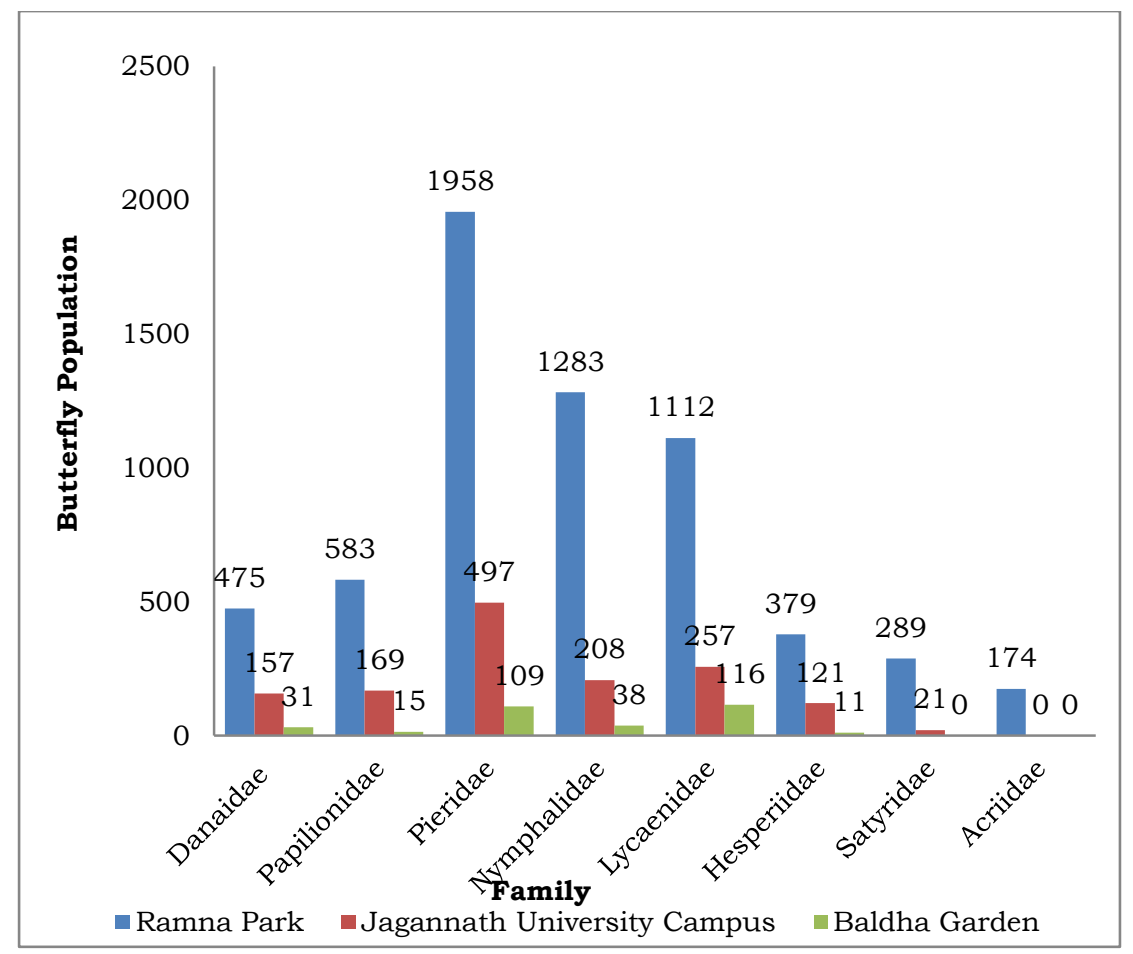

Fig. 5. Family-wise population of butterfly at the three selected areas of the Dhaka city.

The diversity indices $\mathrm{H}$ and $\lambda$ appear useful as it incorporates species richness (Ganeshaih et al. 1997). In this study Shanon's Diversity Index appears to have high value $(\mathrm{H}=3.68$ for the Ramna Park, $\mathrm{H}=3.20$ for the Jagannath University Campus and $\mathrm{H}=2.50$ for the Baldha garden) and Simpson's Index ( $\lambda$ ) gave the 
low value $(\lambda=0.03$ for the Ramna park, $\lambda=0.06$ for the Jagannath University and $\lambda=0.13$ for the Baldha garden) indicating plenteous diversity richness for butterfly species in these three selected areas. These also indicate

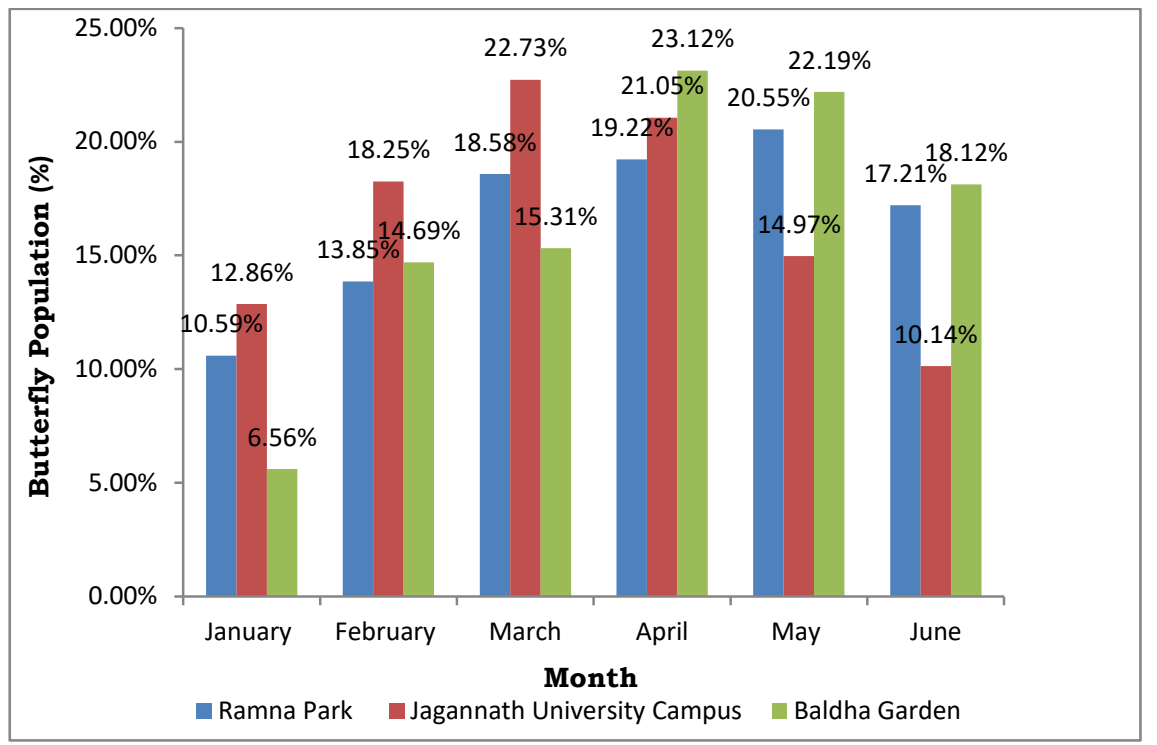

Fig. 6. Month-wise population of butterfly (\%) at the three selected areas of the Dhaka city.

Table 4. Community Similarity among the Ramna Park, Jagannath University Campus and Baldha Garden

Community Similarity is measured by Sorenson's Coefficient (CC).

The equation is: Sorenson's Coefficient $(\mathrm{CC})==\frac{3 \mathrm{C}}{\mathrm{S} 1+\mathrm{S} 2+\mathrm{S} 3}$

Where,

The number of species in the three communities (Ramna Park, Jagannath University Campus and

Baldha Garden) have in common $(C)=17$, The total number of species found in the Ramna Park $\left(\mathrm{S}_{1}\right)$

$=52$, The total number of species found in the Jagannath University Campus $\left(\mathrm{S}_{2}\right)=37$, and the total number of species found in the Baldha Garden $\left(\mathrm{S}_{3}\right)=20$.

Sorenson's Coefficient $(\mathrm{CC})==\frac{3 \mathrm{C}}{\mathrm{S} 1+\mathrm{S} 2+\mathrm{S} 3}=\frac{3 \times 17}{52+37+20}=\frac{51}{109}=0.47$.

that the highest diversity of these three areas is in the Ramna Park, and the lowest is in the Baldha Garden, since the value of $\lambda$ decrease with the increasing diversity (Ludwing and Reynolds 1988). The Shannon-Wiener Index of diversity has demonstrated some sort of contradictory outcome, interference habitat or forest gaps have higher butterfly diversity than that in closed canopy or dense forests (Wood and Gillman 1998). Nidup et al. (2014) described Shannon's Diversity Index 3.16 indicated high butterfly diversity in Royal Monas National Park, Bhutan. Simpson's Index of Diversity of these three areas indicated high 
butterfly diversity of which the topmost diversity was in the Ramna Park, and the lowest was in the Baldha Garden.The Simson's Reciprocal Index is also shown high butterfly diversity of these areas. According to the index values the maximum and minimum diversity was found as same as Simpson's Index of Diversity

Evenness index provides and insight into the relative abundance of the species in the community. The value of $\mathrm{E}$ tend to be zero indicates that the species become more dominant in a community (Sanjayan et al. 1995). In this study, the value of E (0.93) for the Ramna Park is so high while the Jagannath University $(E=0.89)$ and Baldha Garden $(E=0.84)$ also have high value of $E$ that indicates the species are evenly distributed.

Community Similarity among the Ramna Park, Jagannath University Campus and Baldha Garden is shown in Table 4. The value of Sorenson's Coefficient $(\mathrm{CC})$ of these communities is 0.47 . The range of it is from 0 to 1 , the closer the value is to 1 , the more the communities have in common (Sorensen 1948). According to Sorenson's Coefficient these three communities have quite a bit of overlap or similarity.

\section{CONCLUSION}

The present research has revealed butterfly biodiversity and prepared checklists in the study areas. This study revealed 75 butterfly species belongings to 42 genera under 8 major families. On the basis of the data, conservation of wide range of indigenous butterfly fauna in these small human dominated landscapes might be a good model for maintaining most favourable habitat within fragments.

Acknowledgement: The authors are grateful to the Ramna Park, Jagannath University Campus and Baldha Garden authorities for giving permission and support to conduct this work.

\section{LITERATURE CITED}

AHMAD, M., KABIR, S. M. H., AHMED, A. T. A., RAHMAN, A. K. A., AHMED, Z. U., BEGUM, Z. N. T., HASSAN, M. A. and KHONDKER, M. 2009. Encyclopedia of Flora and Fauna of Bangladesh.Asiatic Society of Bangladesh.Dhaka. Vol. 21(III), 460 pp.

ALURI, J. S. R and RAO, S. P. 2002. Psychophily and evolution consideration of Cadaba fructicosa (Capparaceae). J. Bombay Nat. His. Soc. 99(1):59-63.

Bangladesh, I. U. C. N. 2015. Red list of Bangladesh volume 7: Butterflies. IUCN, Bangladesh Country Office, Dhaka. 400 pp.

BASHAR, M. A. 2014. Butterflies of Bangladesh-A broad approach for nature lovers, First Ed. Biodiversity Conservation Trust Foundation Publications, Dhaka. 514 pp. 
BINGHAM, C. T. 1905. The fauna of British India including Ceylon and Burma Butterflies.Taylor and Francis, Red lion court, Fleet Street, Vol. 1, ix + 511 pp.

BLAIR, R. B. 1999. Birds and butterflies along an urban gradient: surrogate taxa for assessing biodiversity? Ecol. appl. 9(1):164-170.

CALDAS, A. and ROBBINS, R. 2003. Modified Pollard transects for assessing tropical butterfly abundance and diversity. Biol. Conserv. 110: 211-219.

EVANS, W. H. 1932. The Identification of Indian Butterflies.Today and Tomorrow's Printers and Publishers, New Delhi, 454 pp.

GANESHAIAH, K. N., CHANDRASHEKARA, K. and KUMAR, A. R. V. 1997. A new measure of biodiversity based on biological heterogeneity of the. Current Science 73(2):128-133.

GAONKAR, H.1996. Butterflies of the Western Ghats, India including Sri Lanka: A biodiversity assessment of a threatened mountain system. Centre for Ecological Sciences, London, 49 pp.

KOIRALA, T. P., KOIRALA, B. K. and KOIRALA, J. 2020. Butterfly diversity in Gidakom Forest Management Unit, Thimphu, Bhutan. J. Threat. Taxa 12(8): 15794-15803.

KUNTE, K. 2000. Butterflies of Peninsular India, University Press Limited Hyderabad India. 254 pp.

LAFONTAINE, J. D. 1997. Butterflies and Moths. In: Assessment of species diversityin the Mixed Wood Plains ecozone. (Ed., Smith, I. M.). Eman Publication, Karachi, Pakistan. pp. 1-31.

LUDWING, J. A. and REYNOLDS, J. F. 1988. Statistical ecology: a primer on methods and computing. John willy and Sons, New York. Vol. 1, 220 pp.

MacDONALD, Z. G., NIELSEN, S. E. and ACORN, J. H. 2017. Negative relationships between species richness and evenness render common diversity indices inadequate for assessing long-term trends in butterfly diversity. Biodiv. Conserv. 26 (3):617-629.

MARSHALl, G. F. L. and DE NICEVILlE, L. 1883. Butterflies of India, Burmah and Ceylon.A. J. Reprints Agency, New Delhi, Vol 1, 338 pp.

NIDUP, T., DORJI, T. and TSHERING, U. 2014. Taxon diversity of butterflies in different habitat types in Royal Manas National Park. Journal of Entomology and Zoology Studies 2(6):292-298.

NIMBALKAR, R. K., CHANDEKAR, S. K. and KHUNTE, S. P. 2011. Butterfly diversity in relation to nectar food plants from Bhor Tahsil, Pune District, Maharashtra, India. J. Threat. Taxa, 3 (3):1601-1609.

PADHEY, A., SHELKE, S. and DAHANUKAR, N. 2012. Distribution and composition of butterfly species along the latitudinal and habitat gradients of the Western Ghats of India. Check List 8(6): 1196-1215.

RAFI, M. A., MATIN, M. A., SHEIKH, M. K. and ASHFAQUE, M. 2000. Papilionid (swallowtails) butterflies of Pakistan.Gul Awan Printers, Islamabad, Pakistan, pp. 1-33.

ROBBINS, R .K. and OPLER, P. A. 1997. Butterfly diversity and a preliminary comparison with bird and mammal diversity. In: Biodiversity II, understanding and protecting our biological 
resources. (Ed., WILSON, D. E., REAKA-KUDLA, M. L. and. WILSON, E. O.) Joseph Henry Press, Washington, DC, pp. 69-82.

SANJAYAN, K. P., MURALIRANGAN, M. C., SURESH, P. D., SURESH, C. and ALBERT, S. 1995. The plant community structure of the Namangalam Reserve Forest, Tamil Nadu: a paradigm of the spatial distribution pattern in a natural scrub-jungle ecosystem. Int. J. Ecol. Env. Sci. 21:297307.

SARDER, R., ALIM, M. A., HABIB, A. H. M. S. and ISLAM, M. A. 2016. Butterflies of Keraniganj.J. Taxon. Biodiv. Res.7: 1-8.

SCHMUCKI, R., PE'ER, G., ROY, D. B., STEFANESCU, C., VAN SWAAY, C. A., OLIVER, T. H., KUUSSAARI, M., VAN STRIEN, A. J., RIES, L., SETTELE, J. and MUSCHE, M. 2016. A regionally informed abundance index for supporting integrative analyses across butterfly monitoring schemes. J. Appl. Ecol. 53(2): 501-510.

SHANNON, C. E. and WEINER, W. 1949. The mathematical theory.University of Illinois press, Urbana.117 pp.

SIMPSON, E.H. 1949. Measurement of diversity. Nature 163: 688.

SINGH, A. P. 2011. Butterflies of India. Om Books Int., Noida. 184 pp.

SORENSEN, T. 1948. A method of stabilizing groups of equal amplitude in plant sociology based on similarity of species content and its application to analysis of vegetation on Danish commons. Biol. Skrifter 5: 1-34.

TALBOT, G. 1978. The Fauna of British India including Ceylone and Barma, Butterflies. Today and Tomorrow's Printers and Publishers, New Delhi, India, Vol. 1, 660 pp.

WHITTAKER, R. H. 1972. Evolution and measurement of species diversity. Taxon 21: 213-251.

WOOD, B., and GILLMAN, M. P. 1998. The effects of disturbance on forest butterflies using two methods of sampling in Trinidad. Biodiv. Conserv. 7(5): 597-616.

WYNTER-BLYTH, M. A. 1957. Butterflies of Indian Region, Bombay Natural History Society, Bombay, $523 \mathrm{pp}$.

(Manuscript received on 15 October, 2020 revised on 3 November, 2020) 